\title{
Efeitos do mindfulness em gestantes: uma revisão sistemática da literatura entre 2010
}

\section{e 2020}

\author{
Effects of mindfulness on pregnant women: a systematic literature review between 2010 and 2020 \\ Efectos de la mindfulness en las mujeres embarazadas: una revisión sistemática de la literatura
}

entre 2010 y 2020

Recebido: 11/10/2021 | Revisado: 18/10/2021 | Aceito: 25/10/2021 | Publicado: 28/10/2021

Anne Carolline Bispo dos Santos ORCID: https://orcid.org/0000-0003-4632-6674 Universidade Tiradentes, Brasil

E-mail: anne.bispo93@gmail.com

Halley Ferraro Oliveira

ORCID: https://orcid.org/0000-0003-0123-7395 Universidade Tiradentes, Brasil

E-mail: halleyoliveira62@gmail.com

Cauane Silva Oliveira Santos

ORCID: https://orcid.org/0000-0002-3896-4476 Universidade Tiradentes, Brasil

E-mail: cauane_96@yahoo.com

Vitória Dias dos Santos

ORCID: https://orcid.org/0000-0001-7350-3678

Universidade Tiradentes, Brasil

E-mail: vitoria.ddos@souunit.com.br

\begin{abstract}
Resumo
Este trabalho teve como objetivo apresentar os principais efeitos do mindfulness em gestantes que estão sendo pesquisados nos últimos 10 anos (2010-2020). Nesse sentido, buscou-se através de uma Revisão Sistemática de Literatura (RSL), recuperar e avaliar estudos científicos que apresentam alguma contribuição no âmbito da avaliação dos principais efeitos dessa terapia alternativa em gestantes, em qual trimestre gestacional essa técnica é mais estudada e qual o risco gestacional (alto ou baixo risco) da maioria das gestantes estudadas. Para isso, os dados foram coletados e organizados a partir das bases de dados da PubMed e BVS (Biblioteca Virtual de Saúde), ambas amplamente aceitas pela academia médica brasileira. Os resultados apontam que os efeitos mais prevalentes nos estudos analisados são os de cunho psicológico, sendo a redução dos sintomas depressivos os principais. Contudo, também foram encontrados efeitos físicos e comportamentais nas gestantes estudadas. Além disso, essas gestantes estavam vivenciando o $2^{\circ}$ trimestre gestacional e eram classificadas em sua maioria como de baixo risco.
\end{abstract}

Palavras-chave: Mindfulness; Meditação; Gestação; Gestantes.

\begin{abstract}
This work aimed to present the main effects of mindfulness in pregnant women that are being researched in the last 10 years (2010-2020). In this sense, we sought, through a Systematic Literature Review (RSL), to retrieve and evaluate scientific studies that present some contribution in the evaluation of the main effects of this alternative therapy in pregnant women, in which gestational trimester this technique is most studied and in which gestational risk (high or low risk) of most pregnant women studied. For this, data were collected and organized from PubMed and BVS (Virtual Health Library) databases, both widely accepted by the Brazilian medical academy. The results show that the most prevalent effects in the studies analyzed are those of a psychological nature, with the reduction of depressive symptoms being the main ones. However, physical and behavioral effects were also found in the studied pregnant women. In addition, these pregnant women were experiencing the 2 nd trimester of pregnancy and were mostly classified as low risk.
\end{abstract}

Keywords: Mindfulness; Meditation; Pregnant; Pregnancy.

\section{Resumen}

Este trabajo tuvo como objetivo presentar los principales efectos del mindfulness en mujeres embarazadas que se están investigando en los últimos 10 años (2010-2020). En este sentido, se buscó, a través de una Revisión Sistemática de la Literatura (RSL), recuperar y evaluar estudios científicos que presenten algún aporte en la evaluación de los principales efectos de esta terapia alternativa en gestantes, en cuyo trimestre gestacional es más estudiada esta técnica. y en los que se estudió el riesgo gestacional (alto o bajo) de la mayoría de las mujeres embarazadas. Para ello, se recopilaron y 
organizaron datos de las bases de datos PubMed y BVS (Virtual Health Library), ambas ampliamente aceptadas por la academia médica brasileña. Los resultados muestran que los efectos más prevalentes en los estudios analizados son los de carácter psicológico, siendo la reducción de los síntomas depresivos los principales. Sin embargo, también se encontraron efectos físicos y de comportamiento en las mujeres embarazadas estudiadas. Además, estas mujeres embarazadas estaban experimentando el segundo trimestre de embarazo y en su mayoría se clasificaron como de bajo riesgo.

Palabras clave: Mindfulness; Meditación; Embarazo; Embarazadas.

\section{Introdução}

Mindfulness é traduzido como "atenção plena", ou seja, o não esquecimento da mente em relação ao objeto experimentado, sendo a sua função a não distração - um conceito que se aproxima do que a neuropsicologia chama de memória de trabalho, ou memória operacional (Cosenza, 2021). O objetivo do mindfulness disposto dessa forma nos ensinamentos mais antigos propõe como a realidade última da prática a busca pela sabedoria e a completa compreensão das características essenciais daquilo que estamos observando, entendendo em seu âmago a real impermanência desses fenômenos, a presença da insatisfatoriedade, a natureza daquilo que se experimenta, com a mente observando a si mesma em todos os aspectos (Souza, 2020).

Diante disso, o Mindfulness é considerado uma das cinco modificações possíveis da mente, sendo atingidos quando o praticante se desliga por completo do mundo exterior, alcançando estágios profundos de concentração. Dessa forma, a memória consolida-se, torna-se clara e pura, fazendo que o conhecimento real do objeto de observação da prática - seja a sua respiração, suas sensações físicas ou um som - brilhe através da mente e, assim, um novo grau de consciência é atingido. Nesse estado, a confusão mental que era comum acontecer na mente vai sendo trocada, aos poucos, por um estado de maior clareza no qual novos níveis de conhecimento e consciência são desenvolvidos (Souza, 2020).

Por fim, a introdução de mindfulness no campo da psicologia, da medicina comportamental e no dia a dia dos ocidentais é relativamente recente. Iniciou nos últimos anos do século XX com o trabalho de Jon Kabat-Zinn no Centro Médico da Universidade de Massachusetts, nos Estados Unidos, em que pacientes afetados pela dor e o estresse eram convidados a serem submetidos ao treinamento da atenção diligente, em grupos, com programa elaborado a partir da própria experiência do pesquisador com as tradições orientais budistas (Vandenberghe, 2006).

Diversos estudos mostram que a gravidez e o pós-parto estão associados a aspectos físicos e masculinos mudanças mentais e são o momento crítico para as mães desenvolverem distúrbios (Forray, 2010). Além disso, o estresse e a ansiedade estão associados ao ganho de peso, o que implica nas recomendações da OMS, já que o ganho de peso está diretamente associado a diabetes gestacional, complicações de parto e parto, e retenção de peso pós-parto. Com isso, a aplicação do mindfulness parece ser bastante efetiva na promoção da autorregulação emocional, em especial quando se trata de emoções negativas (Cosenza, 2021).

Dessa forma, a presente revisão sistemática teve como objetivo primário analisar os principais efeitos do uso da técnica de mindfulness ou atenção plena durante a gestação que foram estudados nos últimos 10 anos (2010 -2020). Nesse sentido estabelecemos os seguintes objetivos específicos: Identificar os efeitos do mindfulness nas gestantes estudadas, identificar em qual período gestacional (baseado em trimestres) houve maior número de estudos e por fim identificar qual o tipo de gestação foi mais estudado (alto ou baixo risco).

\section{Metodologia}

Foi utilizada nesta pesquisa, a abordagem eminentemente quantitativa. De acordo com Moresi (2003), a pesquisa quantitativa tem como objetivo traduzir em números, tanto opiniões, atitudes e preferências quanto comportamentos para que posteriormente possam ser analisadas e classificadas, através do uso de recursos e técnicas estatísticas. 
Nesse sentido, com o intuito de atingir os objetivos específicos referidos na seção anterior, empregou-se na pesquisa em tela os recursos estatísticos provenientes da Revisão Sistemática de Literatura (RSL). Assim, as revisões sistemáticas devem ser executadas seguindo uma estratégia pré-definida, a partir das seguintes etapas principais: desenvolver estratégia de busca que permita sua avaliação e reprodutibilidade por outros pesquisadores; considerar um período específico para a busca; recuperar trabalhos que atendam palavras-chaves pré-determinadas; e, definir de forma clara os critérios de inclusão e exclusão dos trabalhos buscados (Dermeval, Coelho \& Bittencourt, 2020). Seguindo a referida estrutura, apresentaremos nas seções seguintes: a estratégia de busca e seleção dos estudos; os critérios de inclusão e exclusão; o processo de extração dos dados a partir dos estudos selecionados e demonstração de discussão; e, os resultados desta investigação. Por fim, foram apresentadas as considerações finais, conforme as questões de pesquisa levantadas nesta revisão sistemática de literatura.

\subsection{Estratégia de Busca e Seleção}

O objetivo desta revisão sistemática é recuperar e avaliar os estudos científicos que apresentam alguma contribuição na avaliação dos principais efeitos do mindfulness na gestação e em qual fase da gestação houve maior aplicabilidade dessa terapia alternativa. Dessa forma, pretende-se responder à seguinte questão de pesquisa principal: Quais os principais efeitos do mindfulness em gestantes?

Com base na questão de pesquisa (QP) principal acima, outras perguntas mais específicas foram levantadas:

- QP1 - Quais os efeitos do estudo (referente a saúde mental, a saúde física, ou outros)?

- $\quad \mathrm{QP} 2$ - Em qual período gestacional (em trimestres) houve maior número de estudos?

- QP3- Em quais grupos de risco gestacional houveram mais estudos (baixo ou alto risco)?

A partir das referidas questões de pesquisa, foram definidos os critérios de seleção de busca, levando em consideração, exclusivamente, a busca automática, conforme protocolo definido em Dermeval, Coelho \& Bittencourt (2020).

Utilizando uma string de busca, apresentada na Figura 1 e definida a partir de termos de pesquisa relacionados às questões anteriormente citadas (QP1, QP2 e QP3), a consulta automática foi realizada através de duas bases de dados amplamente aceitas pela comunidade médica: PUBMED e BVS.

Figura 1 - String de busca automática da RSL.

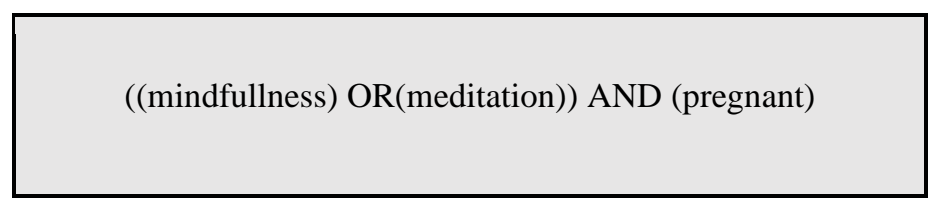

Fonte: Autores.

Dessa forma, a presente pesquisa bibliográfica buscou cobrir os trabalhos mais relevantes da área, no período de 2010 a 2020. A Figura 2 mostra o processo de revisão sistemática e o número de artigos identificados em cada etapa correspondente. 
Figura 2 - Fluxograma de seleção de artigos para composição da RSL.

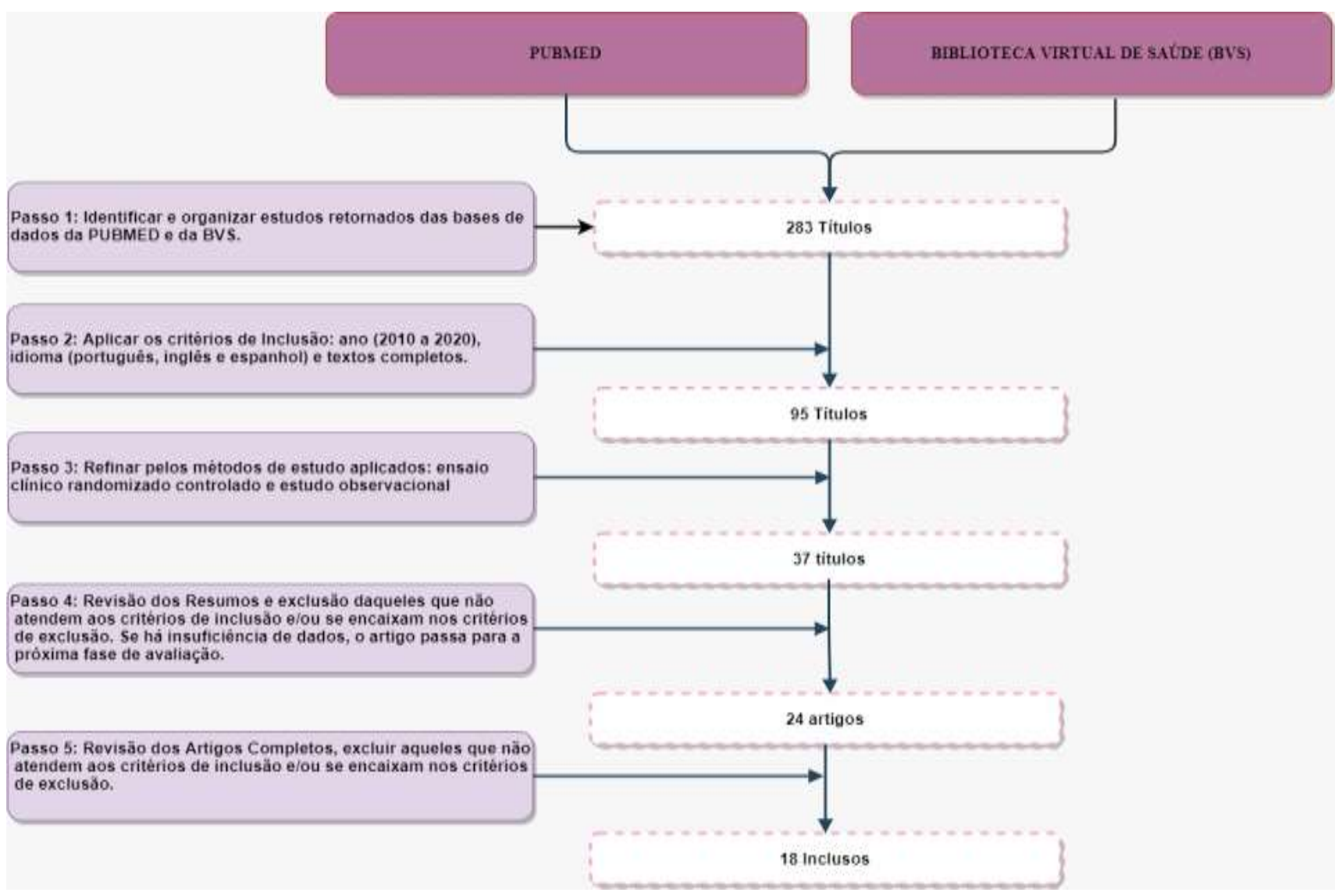

Fonte: Adaptado de Rocha (2014, p. 1266).

Portanto, a estratégia de busca e seleção teve como objetivo capturar os estudos relacionados com os principais aspectos do planejamento participativo no âmbito escolar, sob a perspectiva dos discentes.

2.2 Critérios de Inclusão e ExclusãoDe acordo com Kitchenham e Charters (2007), a definição de critérios de inclusão e exclusão nas revisões sistemáticas tem como objetivo identificar estudos primários que apresentam evidências diretas quanto às questões de pesquisa, além de permitir a redução da probabilidade de viés. Nesse sentido, foram admitidos como critérios de inclusão: Estudos na área da saúde com enfoque na área médica, estudos publicados entre 2010 e 2020, estudos gratuitos ao público, estudos que respondam às questões de pesquisa citadas na Subseção 2.1 deste trabalho; estudos que foquem nos efeitos da utilização do mindfulness durantes a gestação seja ela de baixo ou de alto risco, estudos que foquem na modalidade específica de mindfulness.

Quanto aos critérios de exclusão utilizados, são eles: estudos que foquem em outro tipo de terapia alternativa (yoga, aromaterapia, musicoterapia, acupuntura, etc), estudos publicados antes de 2010 ou depois de 2020; estudos que foram teses de monografias, estudos que estão em capítulos de livros, estudos que não são publicações completas (slides Power Point,resumo, etc.); estudos secundários; estudos redundantes de mesma autoria (considera-se o estudo mais completo, com preferência para publicação mais recente); estudos não relacionados com o tema principal; estudos duplicados.

Os critérios de inclusão e exclusão apresentados foram utilizados em todas as etapas do processo de seleção dos estudos, conforme demonstrado na Figura 2. 


\subsection{Extração dos Dados}

Uma vez efetivada a fase de busca e seleção dos estudos, a extração dos dados se deu através da leitura total de 18 artigos primários selecionados de acordo com os critérios de inclusão e exclusão. Seguindo o modelo de Kitchenham e Charters (2007), desenvolveu-se o formulário de extração apresentado na Tabela 1.

Tabela 1 - Formulário de Extração da RSL.

\begin{tabular}{|c|c|}
\hline Dados de Estudo & Descrição \\
\hline \multicolumn{2}{|l|}{ ID } \\
\hline \multicolumn{2}{|l|}{ Ano } \\
\hline \multicolumn{2}{|l|}{ Título } \\
\hline \multicolumn{2}{|l|}{ Resumo } \\
\hline \multicolumn{2}{|l|}{ Autor } \\
\hline \multicolumn{2}{|l|}{ Referência bibliográfica } \\
\hline \multicolumn{2}{|l|}{ Métodos de estudo } \\
\hline \multicolumn{2}{|l|}{$\begin{array}{c}\text { Atributos relacionados a } Q P 1 \text { - } \\
\text { Efeitos do mindfulness } \\
\end{array}$} \\
\hline \multicolumn{2}{|l|}{$\begin{array}{c}\text { Atributos relacionados a } Q P 2 \text { - } \\
\text { Período gestacional (trimestres de } \\
\text { gestação) }\end{array}$} \\
\hline $\begin{array}{c}\text { Atributos relacionados a } Q P 3- \\
\text { Risco gestacional (alto ou baixo risco) }\end{array}$ & \\
\hline
\end{tabular}

Fonte: Adaptado de Rocha (2014, p. 1267).

A partir deste formulário (Tabela 1) foi possível obter tanto os dados gerais dos estudos, quanto dados específicos no que diz respeito às questões de pesquisa da presente RSL. Assim, serão apresentados a seguir, por meio de gráficos estatísticos e tabulação, os dados coletados bem como sua análise e discussão.

\section{Resultados e Discussão}

Nesta seção, serão discutidos os resultados de acordo com os critérios definidos na Tabela 1. Para facilitar a apresentação dos dados, os estudos selecionados serão referenciados através de um identificador (ID) e podem ser acessados em detalhes no link abaixo:

https://docs.google.com/spreadsheets/d/1jLn7eyERgPqAvOMHfOLynb5Q67n5o9Ho/edit?usp=sharing\&ouid=112489 $622680110371759 \&$ rtpof=true $\&$ sd=true

3.1 Ano de publicaçãoForam analisados estudos no período dos últimos dez anos, ou seja, entre 2010 e 2020 . O ano corrente (2021) não foi considerado, pois se encontra em andamento, não sendo adequado obter resultados de um período não concluído. O ano com maior número de publicações foi 2020 (22,2\%), seguido de 2016, 2019 e 2014 (todos com 16,7\%), depois 2013 e 2017 (ambos com 11,1\%) e por último 2018 (5,6\%), conforme Figura 3: 
Figura 3 - Ano de publicação das pesquisas da RSL.

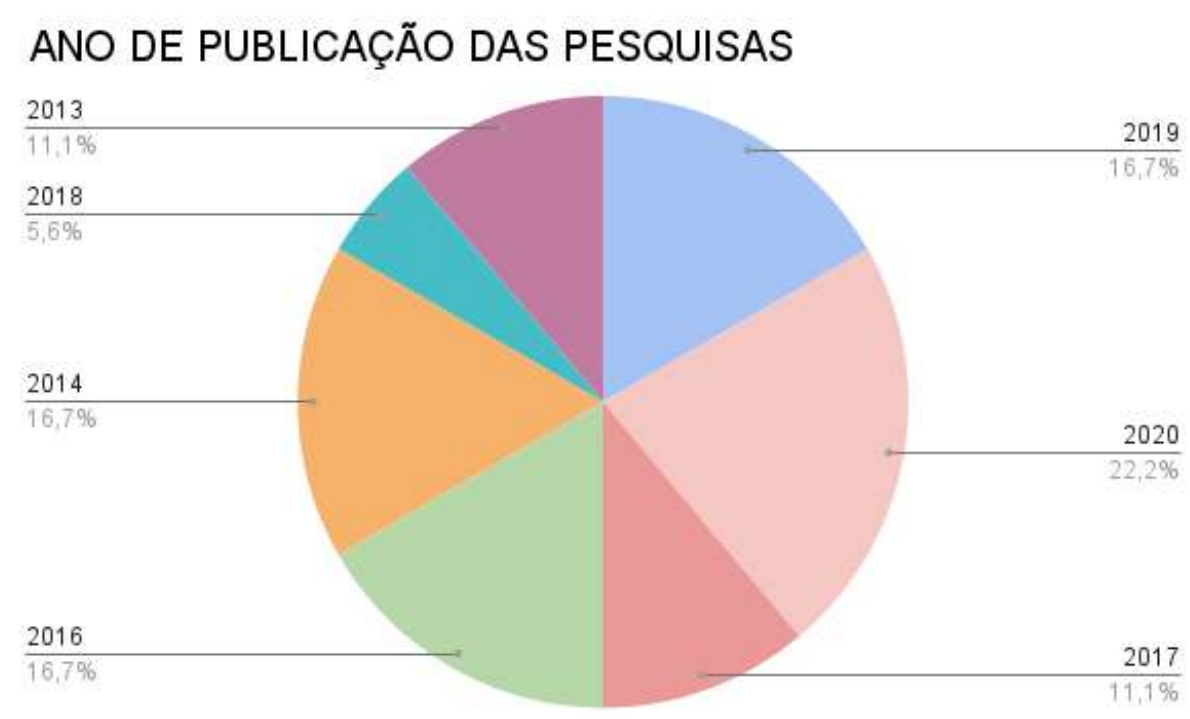

Fonte: Autores.

\subsection{Efeitos do Mindfulness (QP1)}

Esta subseção apresenta os resultados relacionados à QP1, além de discutir as principais implicações de tais resultados. Nesse sentido, o objetivo desta questão de pesquisa foi quais os principais efeitos, sejam eles psicológicos, físicos, ou comportamentais implicados durante o curso da pesquisa com gestantes. A Figura 4 apresenta os resultados encontrados dos efeitos gerais mais prevalentes do mindfulness em gestantes.

Figura 4 - Efeitos gerais do mindfulness nas gestantes da pesquisa da RSL.

\section{EFEITOS DO MINDFULNESS}

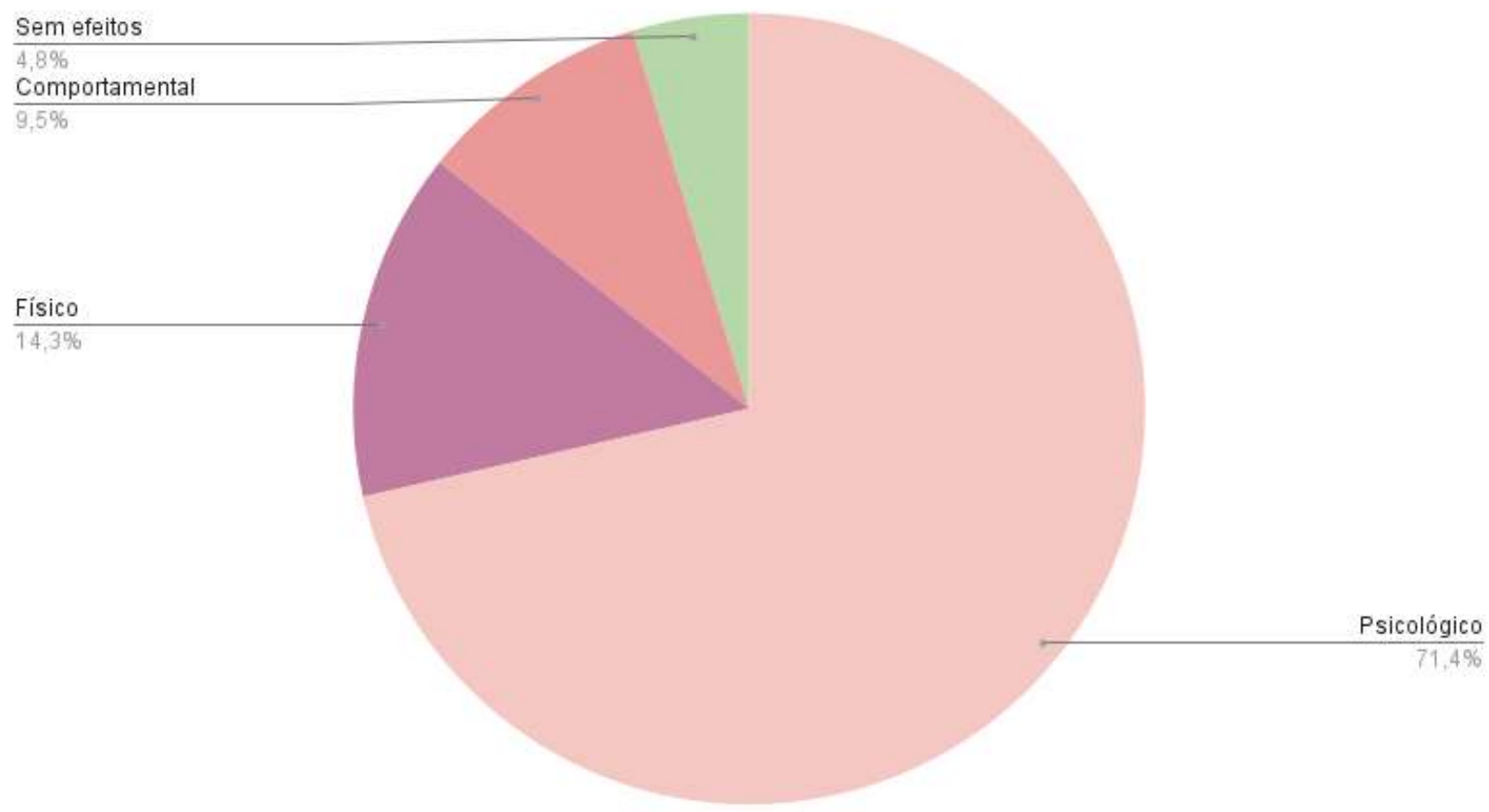

Fonte: Autores. 
De acordo com a Figura 4, observa-se que os efeitos psicológicos nas gestantes foram os mais prevalentes com 71,4\%, seguidos dos efeitos físicos (14,3\%), comportamentais $(9,5 \%)$ e apenas 4,8\% dos estudos não possuíam nenhum efeito aparente. A Figura 5 foi confeccionada com o intuito de mostrar os efeitos específicos do mindfulness nas gestantes estudadas.

Figura 5 - Efeitos específicos do mindfulness nas gestantes da pesquisa da RSL.

\section{EFEITOS ESPECIFICOS DO MINDFUUNESS NA GESTAÇÃO}

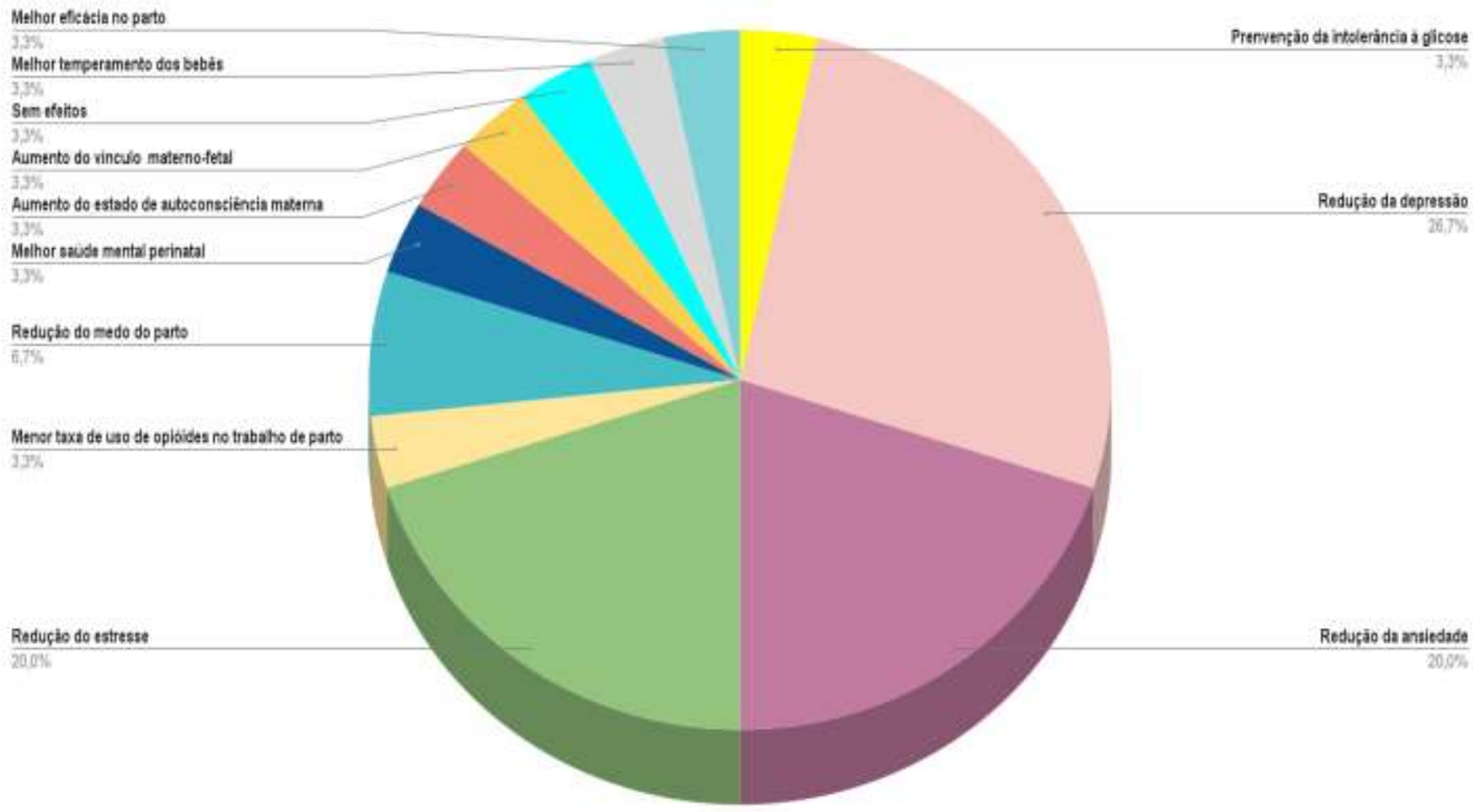

Fonte: Autores.

A Tabela 2 apresenta os resultados de forma mais específica para essa questão de pesquisa, citando diretamente os efeitos encontrados nos estudos, como por exemplo, redução da ansiedade, redução da depressão, redução do estresse do parto, melhora da saúde mental perinatal, aumento da autoconsciência materna, redução do medo do parto (sendo esses classificados como efeitos psicológicos). Além desses, há também os efeitos de cunho comportamentais (aumento do vínculo materno-fetal e melhor temperamento dos bebês) e por último os efeitos físicos (prevenção da intolerância à glicose, menor taxa do uso de opióides durante o parto e melhor eficácia do parto). 
Tabela 2 - Efeitos específicos do mindfulness nas gestantes estudadas na questão de pesquisa 1 da RSL.

\begin{tabular}{|c|c|c|c|}
\hline Efeitos principais & Estudos (ID) & Quantidade & $\%$ \\
\hline Redução da depressão & E01, E03, E04, E05, E11, E12, E15, E18 & 08 & $26,7 \%$ \\
\hline Redução da ansiedade & E02, E07, E09, E12, E17, E18 & 06 & $20 \%$ \\
\hline Redução do estresse & E02, E04, E06, E09, E11, E14 & 06 & $20 \%$ \\
\hline Melhor saúde mental perinatal & E08 & 01 & $3,3 \%$ \\
\hline Aumento da autoconsciência materna & E09 & 01 & $3,3 \%$ \\
\hline Redução do medo do parto & E03, E17 & 02 & $6,7 \%$ \\
\hline Melhor temperamento dos bebês & E16 & 01 & $3,3 \%$ \\
\hline Aumento do vínculo materno fetal & E10 & 01 & $3,3 \%$ \\
\hline Prevenção da intolerância à glicose & E01 & 01 & $3,3 \%$ \\
\hline Menor taxa de uso de opióides no parto & E03 & 01 & $3,3 \%$ \\
\hline Melhor eficácia do parto & E17 & 01 & $3,3 \%$ \\
\hline Não houveram efeitos & E13 & 01 & $3,3 \%$ \\
\hline
\end{tabular}

Fonte: Autores.

Dessa forma, observa-se através da Tabela 2, que a maior parte dos efeitos do mindfulness em gestantes identificada nos estudos selecionados foram de cunho psicológico, sendo a redução da depressão o mais prevalente $(26,7 \%)$, seguidos de redução da ansiedade e do estresse (ambos com 20\%), redução do medo do parto $(6,7 \%)$, melhora da saúde mental perinatal, aumento da autoconsciência materna (ambos com 3,3\%). Dos efeitos comportamentais (aumento do vínculo materno-fetal e melhor temperamento dos bebês), ambos obtiveram as mesmas taxas de 3,3\%. Já os efeitos físicos (prevenção da intolerância à glicose, menor taxa do uso de opióides durante o parto e melhor eficácia do parto) ambos com as mesmas taxas, também de 3,3\%. Em 01 (um) estudo, não foram encontrados efeitos significativos da técnica estudada nas gestantes $(3,3 \%)$.

\subsection{Período Gestacional (QP2)}

Esta subseção apresenta os resultados relacionados à QP2, além de discutir as principais implicações de tais resultados. Nesse sentido, o objetivo desta questão de pesquisa foi identificar a em qual período gestacional a maior parte dos estudos se concentrou. De acordo com o Rezende, a gestação pode ser dividida em três períodos de acordo com suas especificações, são elas o primeiro trimestre (que vai da concepção à 14 semana), segundo trimestre (da $15^{\mathrm{a}}$ à $28^{\mathrm{a}}$ semana) e terceiro trimestre (da $29^{\mathrm{a}}$ à $42^{\circ}$ semana). A Figura 6 a seguir revela os resultados encontrados para a segunda questão de pesquisa. 
Figura 6 - Período gestacional das gestantes estudadas na RSL.

\section{PERIODO GESTACIONAL}

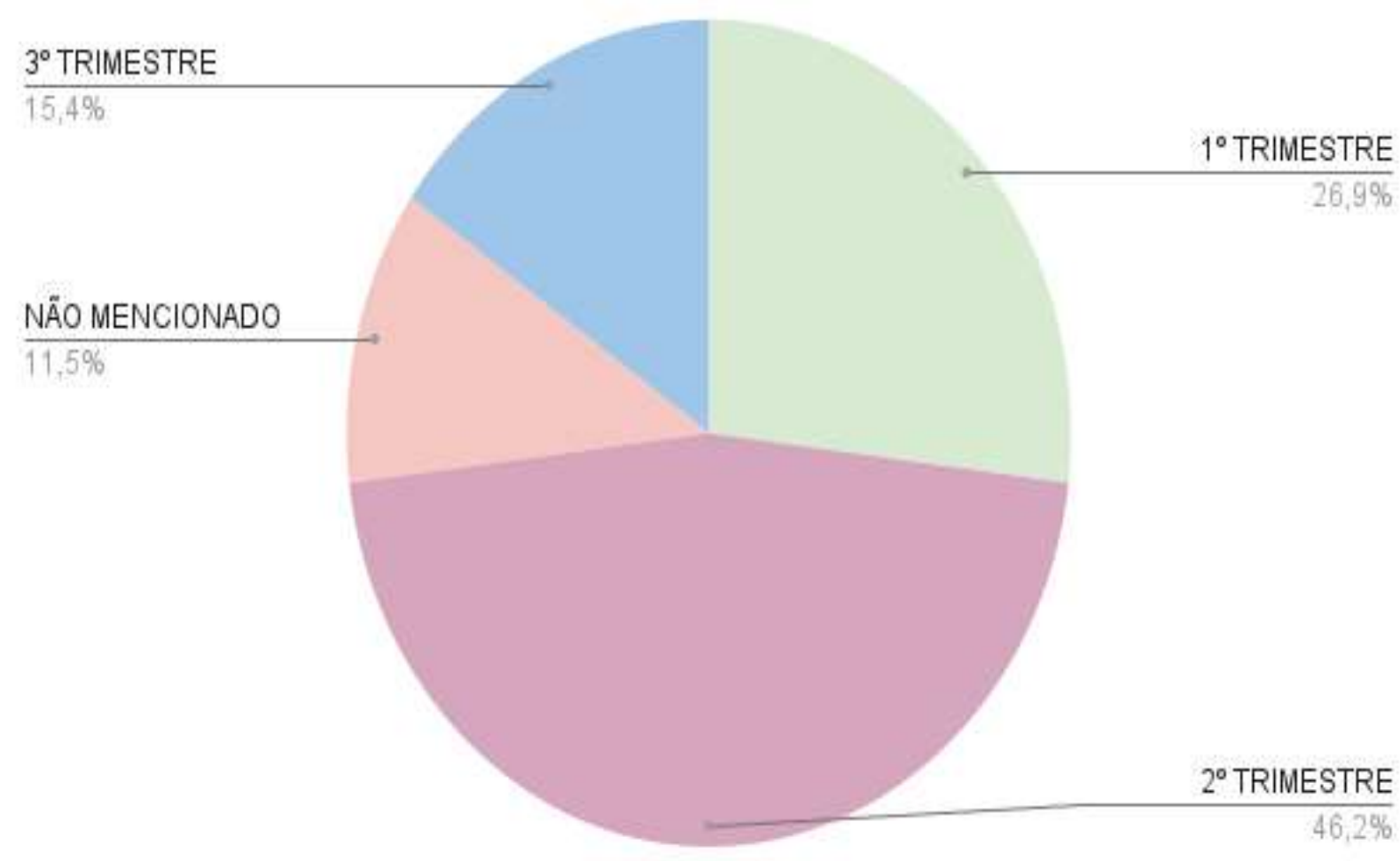

Fonte: Autores.

Dessa forma, a partir da análise dos estudos selecionados, foi-se observado por essa RSL que os estudos se concentraram no segundo trimestre gestacional (com 46,2\% dos estudos), seguidos do primeiro trimestre (26,9\%) e do terceiro (15,4\%). Em alguns estudos $(11,5 \%)$ não foram mencionados ou não foram estudados esse tópico da pesquisa, conforme a Tabela 3 :

Tabela 3 - Período Gestacional na questão de pesquisa 2 da RSL.

\begin{tabular}{|llcr|}
\hline Risco Gestacional & \multicolumn{1}{c}{ Estudos (ID) } & Quantidade & \% \\
\hline $1^{\mathbf{0}}$ Trimestre & E01, E05, E06, E09, E14, E16, E18 & 07 & $26,9 \%$ \\
\hline $2^{\mathbf{0}}$ Trimestre & E01, E04, E05, E06, E07, E09, E10, E11, E12, E16, E17, E18 & 12 & $46,2 \%$ \\
\hline $3^{\circ}$ Trimestre & E03, E05, E07, E08 & 04 & $15,4 \%$ \\
\hline Não mencionado & E02, E13, E15 & 03 & $11,5 \%$ \\
\hline
\end{tabular}

Fonte: Autores.

Dessa forma uma maior concentração de estudos realizados no $2^{\circ}$ Trimestre gestacional pode estar ligado a segurança dos pesquisadores em obter resultados mais expressivos quanto aos benefícios do mindfuness neste período.

\subsection{Risco Gestacional (QP3)}

Esta subseção apresenta os resultados relacionados à QP3, além de discutir as principais implicações de tais resultados. Objetivou-se com essa questão de pesquisa investigar o quais grupos de gestantes foram mais estudados. Nesse sentido, três categorias foram implicadas de acordo os cadernos de atenção às gestantes retirados de Saúde (2012): gestantes de alto risco, 
gestantes de baixo risco. Há também a categoria em que o risco gestacional não foi mencionado no estudo. As categorias estão elencadas conforme Figura 7.

Figura 7 - Risco gestacional das gestantes estudadas na RSL.

\section{RISCO GESTACIONAL}

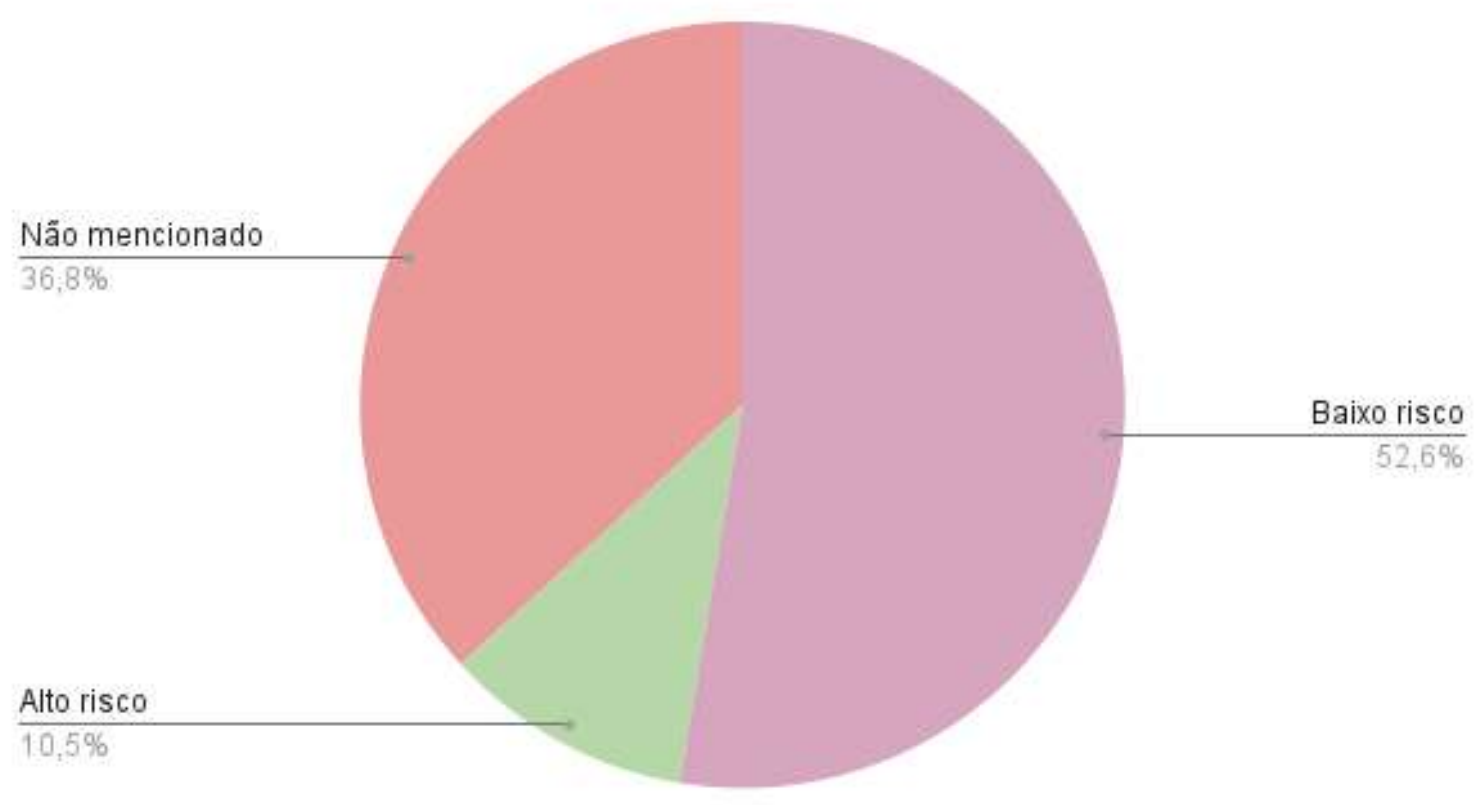

Fonte: Autores.

De acordo com o gráfico, observa-se que as gestações de baixo risco foram mais estudas, com 52,6\%. As gestações de alto risco compreenderam apenas $10,5 \%$ dos estudos e boa parte dos trabalhos não mencionavam qual o risco gestacional das participantes (36,8\%). A Tabela 4 apresenta os resultados de forma mais específica para essa questão de pesquisa.

Tabela 4 - Risco gestacional na questão de pesquisa 3 da RSL.

\begin{tabular}{|llcc|}
\hline Risco Gestacional & Estudos $($ ID $)$ & Quantidade & \% \\
\hline Não mencionado & E04, E06, E09, E11, E15, E17, E18 & 07 & $36,8 \%$ \\
\hline Baixo Risco & E01, E03, E05, E07, E08, E10,E12, E13, E14, E16 & 10 & $52,6 \%$ \\
\hline Alto Risco & E02,E16 & 02 & $10,5 \% \%$ \\
\hline
\end{tabular}

Fonte: Autores.

Observa-se, portanto, que a maior prevalência de estudos em gestantes de baixo risco, pode estar relacionado a uma falta de segurança em se aplicar essa técnica relativamente nova em gestantes com possibilidades de complicação no decorrer da pesquisa.

\section{Considerações Finais}

O objetivo desta revisão sistemática de literatura foi levantar as pesquisas existentes que apresentam alguma contribuição para a avaliação dos efeitos do mindfulness em gestantes. Para cumprir o referido objetivo a pesquisa buscou responder a três questões específicas de pesquisa (QP1, QP2 e QP3). 
Respondendo a QP1, os resultados demonstram que os principais efeitos do mindfulness em gestantes são predominantemente de cunho psicológico, mais especificamente de redução da depressão, sendo referenciado por 08 estudos $(26,7 \%)$.

Quanto à QP2, identificou-se que 12 estudos $(46,2 \%)$ tiveram como participantes gestantes do $2^{\circ}$ trimestre gestacional, período que vai da $15^{\mathrm{a}}$ semana até a $28^{\circ}$ semana de gestação.

Por fim, respondendo a QP3, foram avaliadas gestantes com risco gestacional baixo, sendo contabilizados 10 estudos $(52,6 \%)$.

A predominância dos estudos em gestantes de baixo risco pode estar ligada ao receio que ainda existe entre os pesquisadores de expor gestantes de um risco mais elevado a uma prática alternativa que ainda não se configura como realmente efetiva ou segura na área médica.

Ainda com relação à QP3, essa revisão demonstra uma carência de estudos ou de menção do risco gestacional nos estudos analisados. É importante que os novos estudos tragam de forma clara esse aspecto tão relevante tanto na teoria como na prática da Obstetrícia.

Portanto, evidencia-se a necessidade do desenvolvimento de mais pesquisas que avaliem gestantes de alto risco gestacional e que façam um panorama dos efeitos durante todos os períodos gestacionais. Dessa maneira, a real eficácia do mindfulness poderá ser obtido e aplicada nas gestantes.

\section{Referências}

Byrne J., Hauck Y., Fisher C., Bayes S., \& Schutze R. (2014) Effectiveness of a Mindfulness-Based Childbirth Education pilot study on maternal self-efficacy and fear of childbirth. J Midwifery Womens Health. 59(2):192-7.

Chan K. P. (2014). Prenatal meditation influences infant behaviors. Infant Behav Dev. 37(4):556-61. 10.1016/j.infbeh.2014.06.011.

Cosenza, R. M. (2021). Neurociência e Mindfulness: Meditação, Equilíbrio Emocional e Redução do Estresse. https://integrada.minha biblioteca.com.br/\#/books/9786558820062/.

Dermeval, D., Coelho, Jorge A. P. \& Bittencourt. (2020). Mapeamento Sistemático e Revisão Sistemática da Literatura em Informática na Educação. In: Jaques.

Dimidjian S., Goodman S. H., Felder J. N., Gallop R., Brown A. P. \& Beck A. (2015). An open trial of mindfulness-based cognitive therapy for the prevention of perinatal depressive relapse/recurrence. Arch Womens Ment Health. 18(1):85-94. 10.1007/s00737-014-0468-x.

Dimidjian S., Goodman S. H., Felder J. N., Gallop R., Brown A. P. \& Beck A. (2016). Staying well during pregnancy and the postpartum: A pilot randomized trial of mindfulness-based cognitive therapy for the prevention of depressive relapse/recurrence. J Consult Clin Psychol. 84(2):134-45. 10.1037/ccp0000068.

Duncan, Larissa, G. (2017). Benefits of preparing for childbirth with mindfulness training: a randomized controlled trial with active comparison. BMC pregnancy and childbirth, 17(1), 1-11.

Epel E., Laraia B., Coleman K., Leung C., Vieten C., Mellin L, Kristeller J.L., Thomas M., Stotland N., Bush N., Lustig R.H., Dallman M., Hecht F.M. \& Adler N. (2019). Effects of a Mindfulness-Based Intervention on Distress, Weight Gain, and Glucose Control for Pregnant Low-Income Women: A Quasi-Experimental Trial Using the ORBIT Model. Int J Behav Med. 26(5):461-473. 10.1007/s12529-019-09779-2.

Forray, A. (2010). Onset and exacerbation of obsessive-compulsive disorder in pregnancy and the postpartum period. The Journal of clinical psychiatry, 71(8), $0-0$.

Germer, C. K., Siegel, R. D. \& Fulton, P. R. F. (2016). Mindfulness e Psicoterapia. https://integrada.minhabiblioteca.com.br/\#/books/9788582712443/.

Goetz, M. (2020). Effects of a Brief Electronic Mindfulness-Based Intervention on Relieving Prenatal Depression and Anxiety in Hospitalized High-Risk Pregnant Women: Exploratory Pilot Study. Journal of Medical Internet Research, 22(8), e17593.

Guardino C. M, Dunkel S. C, Bower J. E, Lu M. C \& Smalley S. L. (2014). Randomised controlled pilot trial of mindfulness training for stress reduction during pregnancy. Psychol Health.29(3):334-49. 10.1080/08870446.2013.852670.

Krusche A., Dymond M., Murphy S. E. \& Crane C. (2018). Mindfulness for pregnancy: A randomised controlled study of online mindfulness during pregnancy. Midwifery.65:51-57. 10.1016/j.midw.2018.07.005.

Saúde, M. D. (2012). Secretaria de Atenção à Saúde. Departamento de Atenção Básica. Atenção ao pré-natal de baixo risco. Editora do Ministério da Saúde, 2012

Moresi, E. (2003). Metodologia da pesquisa. Brasília: Universidade Católica de Brasília, 108, 24. 
Research, Society and Development, v. 10, n. 14, e128101421659, 2021

(CC BY 4.0) | ISSN 2525-3409 | DOI: http://dx.doi.org/10.33448/rsd-v10i14.21659

Muthukrishnan S., Jain R., Kohli S. \& Batra S. (2016). Effect of Mindfulness Meditation on Perceived Stress Scores and Autonomic Function Tests of Pregnant Indian Women. J Clin Diagn Res. 10(4):CC05-8. 10.7860/JCDR/2016/16463.7679.

Pan W.L., Chang C. W., Chen S. M. \& Gau M. L. (2019). Assessing the effectiveness of mindfulness-based programs on mental health during pregnancy and early motherhood - a randomized control trial. BMC Pregnancy Childbirth.19(1):346. 10.1186/s12884-019-2503-4.

Rezende J. \& Montenegro C. A. B. (2013). Obstetrícia. (12a ed.) Guanabara Koogan. 186-209.

Sbrilli M. D, Duncan L. G. \& Laurent H. K. (2020). Effects of prenatal mindfulness-based childbirth education on child-bearers' trajectories of distress: a randomized control trial. BMC Pregnancy Childbirth. 20(1):623. 10.1186/s12884-020-03318-8.

Souza, I. C. W. D. (2020). Mindfulness e terapia cognitivo-comportamental. Editora Manole. https://integrada.minhabibliotec a.com.br/\#/books/9 $786555760330 /$.

Tomfohr L. M., Campbell T. S., Giesbrecht G. F., Letourneau N. L., Carlson L. E., Madsen J. W. \& Dimidjian S. (2016). Mindfulness-based cognitive therapy for psychological distress in pregnancy: study protocol for a randomized controlled trial. Trials. 17(1):498. 10.1186/s13063-016-1601-0. PMID: 27737714;

Vandenberghe, L. \& Sousa A. C. A. (2006). Mindfulness nas terapias cognitivas e comportamentais. Rev. Brasileira de Terapia Cognitiva, 2(1), 35-44. <http://pepsic.bvsalud.org/scielo.php?script=sci_arttext\&pid=S1808-56872006000100004\&lng=pt\&nrm=iso〉.

Yang M., Jia G., Sun S., Ye C., Zhang R. \& Yu X. Effects of an Online Mindfulness Intervention Focusing on Attention Monitoring and Acceptance in Pregnant Women: A Randomized Controlled Trial. J Midwifery Womens Health. 2019 Jan;64(1):68-77. 10.1111/jmwh.12944.

Zarenejad M., Yazdkhasti M., Rahimzadeh M., Mehdizadeh T. Z., Esmaelzadeh S. S. (2020). The effect of mindfulness-based stress reduction on maternal anxiety and self-efficacy: A randomized controlled trial. Brain Behav. 10(4):e01561. 10.1002/brb3.1561 